\title{
POLYCRYSTALLINE DIAMOND-ON-POLYMER MICROELECTRODE ARRAYS FOR MECHANICALLY-FLEXIBLE NEURAL INTERFACING
}

\author{
A. Hess ${ }^{1}$, D.M. Sabens ${ }^{2}$, H.B. Martin ${ }^{2}$, and C.A. Zorman ${ }^{1}$ \\ ${ }^{1}$ Department of Electrical Engineering and Computer Science, Case Western Reserve University \\ ${ }^{2}$ Department of Chemical Engineering, Case Western Reserve University, Cleveland, Ohio, USA
}

\begin{abstract}
This paper reports the development of mechanically-flexible, polycrystalline diamond-on-polymer electrodes for use as neural interfaces. Boron-doped diamond has a number of properties that are attractive for use as electrodes in neural recording, stimulation, and electrochemical sensing. However, because the growth of polycrystalline diamond by chemical vapor deposition requires high temperatures $\left(>800^{\circ} \mathrm{C}\right)$, diamond thin films cannot be directly deposited on polymer substrates. To address this limitation, a transfer process was developed to incorporate diamond electrodes onto a polymer substrate. It was demonstrated that the diamondon-polymer devices made using this transfer process retain the mechanical properties of the polymer. Electrical characterization showed that the diamond electrodes have an impedance of $\sim 2-3$ $\mathrm{M} \Omega$ at $1 \mathrm{kHz}$. This and other tests indicate that the diamond-onpolymer electrodes have suitable characteristics for neural recording and stimulation.
\end{abstract}

\section{INTRODUCTION}

Microelectrode arrays for neural stimulation and recording at single and multiple contact points have been successfully fabricated on both silicon and polymer substrates. Flexible polymer substrates offer the advantages of conformability to peripheral neural structures and reduced strain on cortical tissue due to movement [1]. Common electrode materials include gold, platinum, tungsten, titanium nitride, and iridium oxide [2]. These conductive materials are typically deposited by sputtering or evaporation, allowing for straightforward monolithic processing with most substrates, especially temperature-sensitive polymers.

Polycrystalline, boron-doped diamond is an alternative electrode material that has been successfully used for both neural recording [3,4] and stimulation of single neurons [3]. Diamond is also an exceptional electrochemical sensing material, capable of detecting neurotransmitters, such as dopamine and serotonin. The release of these molecules is one way in which neurons communicate. As such, in-situ monitoring of these chemicals is highly desirable.

The distinctive set of properties that make diamond a particularly good electrode are due to its structure and unique surface chemistry. Diamond is a mechanically robust, hard and stiff material with a Young's modulus of $\sim 1000 \mathrm{GPa}$ arising from its $\mathrm{sp}^{3} \mathrm{C}-\mathrm{C}$ bonding structure [7]. Diamond is also chemically stable, as evidenced by its resistance to corrosion, which ensures that it will not degrade in the biological environment and that its surface will not become contaminated by fouling [8]. Diamond's wide potential water window $(-1.25 /+2.3 \mathrm{~V})$ [9] is advantageous for stimulation applications because it permits larger electrode potential excursions without hydrolyzing water and potentially damaging both neural tissue and the electrode itself. Further, the wide water window enables a larger number of analytes to be detected and studied using electrochemical techniques [9]. A high analyte sensitivity for electrochemical sensing is provided by diamond due to its low background current [3,9].

Chemical vapor deposition (CVD) is the typical method used to grow polycrystalline diamond. CVD generally requires the substrate to be exposed to temperatures $>800^{\circ} \mathrm{C}$. Diamond electrodes have previously been grown on high temperature substrates like tungsten wires [3] and silicon wafers [4]. Unfortunately, polymers are incompatible with the high temperatures required for microcrystalline diamond growth, preventing direct monolithic processing of boron-doped, polycrystalline diamond electrodes on a polymer substrate. To address this issue, we have developed a novel "diamond-first" fabrication process to create diamond-on-polymer structures suitable for mechanically-flexible, polycrystalline diamond-based, neural microelectrode arrays capable of recording and stimulation, as well as electrochemical recording.

\section{DEVICE FABRICATION}

To enable the integration of diamond electrodes onto a polymer substrate, the transfer process shown in Figure 1, in which the diamond components are grown on a Si handle substrate prior to incorporating thermally-sensitive polymers in the fabrication sequence, was used. This allows the diamond growth process to be performed at preferred temperatures, enabling high quality diamond growth without damaging the polymer layers.

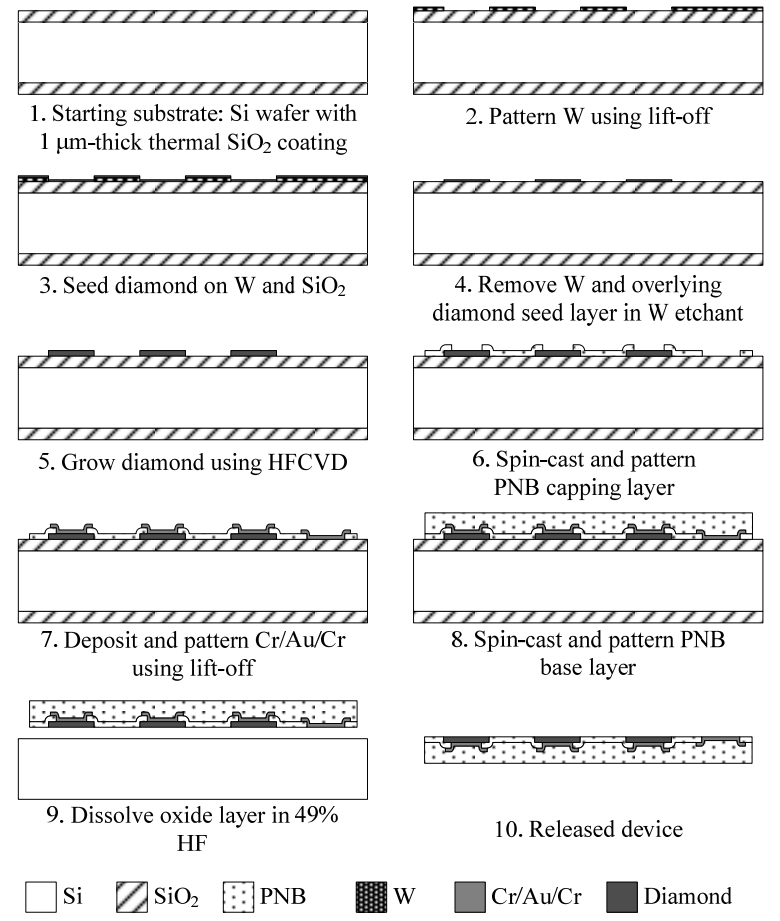

Figure 1: Cross-sectional views of the diamond on polymer electrode array fabrication process

The process begins with the selective growth of diamond thin films. In specific, a 1 to $2 \mu \mathrm{m}$-thick boron-doped diamond layer was selectively grown on a 3 " Si wafer coated with a $1 \mu \mathrm{m}$-thick thermal $\mathrm{SiO}_{2}$ film. A metal template defining the diamond electrodes was patterned on the $\mathrm{SiO}_{2}$ by first spin-casting and patterning a $1.3 \mu \mathrm{m}$-thick Shipley 1813 photoresist film. Next, a 
$100 \mathrm{~nm}$-thick tungsten layer was sputter-deposited and patterned using a lift-off process in acetone with ultrasonic agitation. The wafer was then seeded by sonication in $50 \mathrm{~mL}$ of deionized water containing $20 \mathrm{mg}$ of 1 to $2 \mu \mathrm{m}$ diamond powder, followed by sonication-aided cleaning in pure ethanol. The wafer was then loaded into a hot filament chemical vapor deposition (HFCVD) reactor for a two hour growth process. The filament temperature was $2000^{\circ} \mathrm{C}$, as measured by dual emission optical pyrometry. Pressure was held at 20 Torr with flow rates of $196 \mathrm{sccm}, 1.8 \mathrm{sccm}$ and $4 \mathrm{sccm}$ for hydrogen, methane and $0.01 \%$ trimethylboron in hydrogen, respectively. After the initial growth phase, samples were removed and exposed to a tungsten etch $(10 \%$ sulfuric acid, $10 \%$ hydrogen peroxide in water). After etching, samples were cleaned using a two step sonication process, first in ethanol followed by acetone. The wafer was loaded into the reactor for an additional 4 hours of HFCVD under the conditions described above to selectively grow diamond to a thickness of 1 to $2 \mu \mathrm{m}$. In this final CVD step, diamond growth was limited to the areas with an established diamond seed layer. Diamond does not grow in the field region that had been coated with tungsten.

Next, the first of two polymer layers was processed on the wafer. A $1 \mu \mathrm{m}$-thick polynorbornene (PNB) "capping" layer was spin-cast at $1000 \mathrm{rpm}$ for 30 seconds and soft-baked for 5 minutes at $120^{\circ} \mathrm{C}$ on a hotplate. The capping layer was then patterned with UV radiation $(365 \mathrm{~nm})$ at a dose of $288 \mathrm{~mJ}$, followed by a postexposure bake for 4 minutes at $90^{\circ} \mathrm{C}$ on a hotplate. The capping layer was spray-developed at $2000 \mathrm{rpm}$ using methyl n-amyl ketone (MAK) for 5 seconds and rinsed using PGMEA for 5 seconds. The layer was then cured in a furnace at $160^{\circ} \mathrm{C}$ for 1 hour.

Following the capping layer formation, metal interconnects and connector contact pads were patterned using a lift-off process. A $3.5 \mu$ m-thick layer of AZ nLOF 2035 negative-tone photoresist was spin-cast, soft-baked at $110^{\circ} \mathrm{C}$ for 60 seconds, and defined by $365 \mathrm{~nm}$ UV radiation at a dose $80 \mathrm{~mJ}$. The resist was then baked at $110^{\circ} \mathrm{C}$ for 60 seconds and immersion-developed for 2 minutes in AZ 300 MIF. A three-layer metal combination consisting of 20 nm-thick $\mathrm{Cr} / 250 \mathrm{~nm}$-thick $\mathrm{Au} / 20 \mathrm{~nm}$-thick $\mathrm{Cr}$ was then sputterdeposited and patterned by lift-off. The lift-off step was performed in AZ EBR 70/30 with ultrasonic agitation to dissolve the photoresist and remove all excess metal. The two $\mathrm{Cr}$ layers serve to promote adhesion between the Au layer and the polymer films. Prior to metal deposition, the PNB capping layer was exposed to a 90 second RF preclean step to improve metal-polymer adhesion.

Deposition of the "base" polymer layer comprises the final structural layer of the fabrication process. A $50 \mu \mathrm{m}$-thick PNB layer was spin-coated at 800 RPM for 10 seconds, then 1000 RPM for 30 seconds. The base layer was then soft-baked on a hotplate for five minutes at $120^{\circ} \mathrm{C}$, then defined using i-line UV light at a dose of $2000 \mathrm{~mJ}$. The film was then baked on a hotplate at $90^{\circ} \mathrm{C}$ for 4 minutes, then spray-developed for 90 seconds with MAK rinsed with PGMEA. Finally, the base layer was cured in a furnace at $160^{\circ} \mathrm{C}$ for 1 hour.

The devices were released from the wafer by dissolving the $\mathrm{SiO}_{2}$ sacrificial layer in $49 \% \mathrm{HF}$, exposing the diamond electrode pads from below the polymer and metal layers. The release was performed by applying HF droplets first to the diamond electrode end of the device, then to the device shank, and finally to the connector end of the device. This droplet technique allowed for controlled device removal from the wafer, avoiding damage due to incomplete release at the electrode end of the devices.

In the transfer of the diamond electrodes from the Si substrate to the polymer substrate, the nucleation surface of the diamond becomes the exposed top surface of the electrode, while the growth surface becomes the interface to the metal interconnects. To the best of our knowledge, this is the first time that the nucleation surface has been used as a diamond electrode.

\section{DEVICE DESIGN}

A commercially-available, PNB-based polymer (Avatrel $^{\mathrm{TM}}$ $2585 \mathrm{P}$ ) was chosen as the substrate and capping layers of the diamond-on-polymer devices. Avatrel ${ }^{\mathrm{TM}}$ is attractive because it is spin-castable, photodefinable, and is resistant to moisture absorption and the wet chemical etchants typically used in microfabrication processes. Spin casting allows the solution-based polymer to conform to the diamond sidewalls and faceted growth surface prior to curing, and the chemical resistance ensures device survival during the HF-based release step. The fabrication process is not limited to PNB for the base and capping layers, but does require a material that can conform to the diamond to ensure good adhesion between diamond electrodes and transfer substrate, and survive the release step.

Devices designed for two distinct neural interface applications were fabricated using the diamond transfer technique. A corticalprobe style structure is shown in Figure 2. These devices are designed to be inserted directly into the cortex. These devices have two diamond contacts: an oval electrode with an area of $224 \mu \mathrm{m}^{2}$, and a triangular electrode $1644 \mu \mathrm{m}^{2}$ in area. The electrodes are near the tip of a $2 \mathrm{~mm}$-long, $50 \mu \mathrm{m}$-wide shank. Windows through the capping layer expose two large metal contact pads on the $1 \mathrm{x} 1$ $\mathrm{mm}^{2} \mathrm{tab}$ at the back-end of the device. Connection to external electronics can be made through a wired connection to these pads via conductive epoxy or wirebond. Metal traces $10 \mu \mathrm{m}$ in width connect the diamond electrodes to the contact pads. A via in the capping layer under the diamond electrode enables contact between the diamond electrode and underlying metal trace.

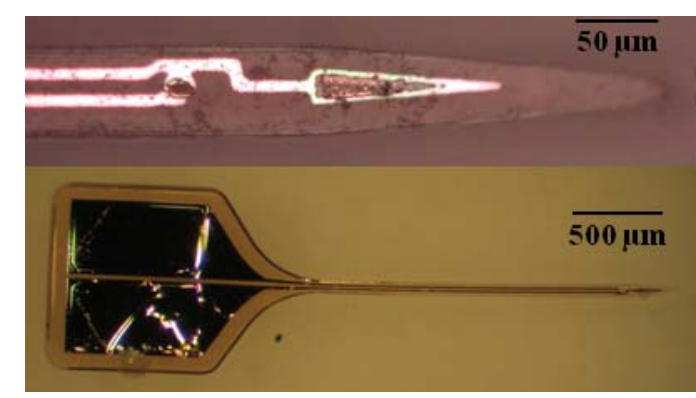

Figure 2: Plan view images of a diamond-on-polymer penetrating cortical probe: (top) close-up of diamond electrodes; (bottom) global view.

The second design, shown in Figure 3, represents a much larger device, better suited for surface contact interfacing with peripheral nerves. Each array is comprised of ten $64 \times 64 \mu \mathrm{m}^{2}$ diamond electrodes at one end, ten corresponding metal contact pads on the other end, with a flexible shaft approximately $2.7 \mathrm{~cm}$ in length and $1.3 \mathrm{~mm}$ in width to support ten $30 \mu \mathrm{m}$-wide metal traces connecting the two ends. Vias in the capping layer underneath the diamond electrodes define the metal-diamond contact area. The connector end is designed to interface with a commercial flexible circuit connector (Hirose ${ }^{\mathrm{TM}} \mathrm{FH}-19$ ).

A variation of the second design (not shown) has been developed for applications requiring an electrode with a large surface area. Due to the rigidity of the diamond films, flexing would induce cracking in large diamond electrodes. Therefore, the larger electrodes were divided into ten electrically connected diamond segments, with each segment consisting of one 64 x 64 $\mu \mathrm{m}^{2}$ diamond pad and $64 \mu \mathrm{m}$ separating the diamond segments. 
The effective area of the electrode is thus increased by a factor of ten, while retaining the flexibility of the device.

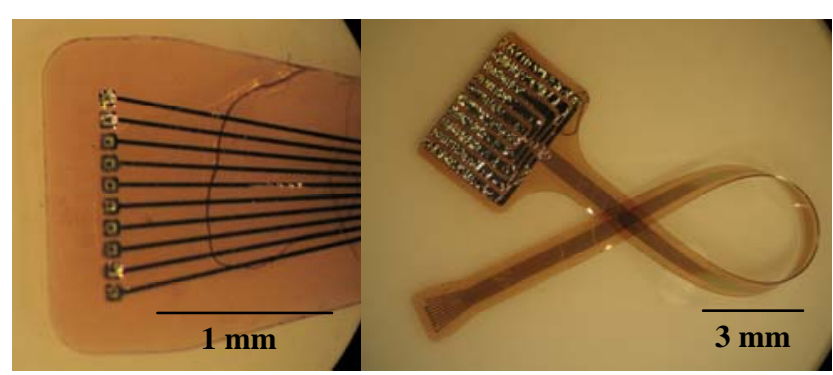

Figure 3: Completed diamond electrode array: (left) close-up of the diamond electrode array with associated metal interconnects; (right) global view showing the flexibility of the device

\section{MECHANICAL CHARACTERIZATION}

The mechanical durability of the diamond-on-polymer structures was investigated using the devices shown in Figure 4. Flexibility was demonstrated through the use of the dog-boneshaped structures, which included eight $100 \mu \mathrm{m}$-diameter diamond electrodes equally distributed along the length of the beam. The devices were wrapped around a cylinder with a radius of $\sim 650 \mu \mathrm{m}$ and observed for delamination or cracking as a resulting of the flexing. No deformation of the diamond or polymer was detected, indicating that the flexibility of the polymer substrate was preserved.

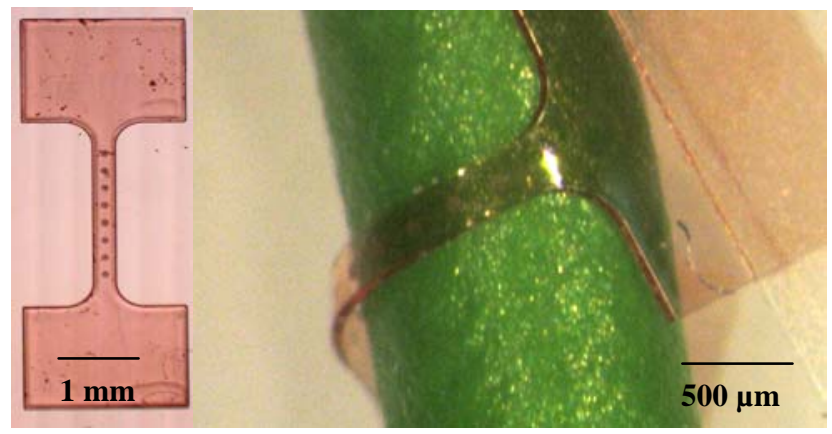

Figure 4: (left) Dog-bone structure with embedded diamond electrodes, and (right) flexed structure.

Further mechanical characterization was performed using a custom-built microtensile tester described in detail in [9]. One pad of the dog-bone structure was placed in a grip that was mounted to a linear piezomotor drive rod, while the other pad was held in a fixed position. As the beam was stretched by movement of the piezomotor, the strain induced in the sample was measured using a digital indicator, and the force on the beam was measured with a load cell. The motor, indicator, and load cell were all computercontrolled using data acquisition software.

Devices with eight $100 \times 100 \mu \mathrm{m}$ square diamond pads along the length of the beam were tested using the microtensile tester by pulling to fracture, and results were compared to the stress-strain plots from PNB-only samples. It was found that the Young's modulus, as determined by the slope in the linear region of the stress-strain plot, of the samples with diamond closely matched that of the PNB-only sample, which was $\sim 800 \mathrm{MPa}$. This value is consistent with the reported value of Young's modulus for Avatrel $^{\mathrm{TM}}$ 2585P. The stress-strain curve for the device with diamond pads is shown in Figure 5. It can be seen that the device remains in the elastic region until approximately $2.4 \%$ strain, at which point the elastic limit is reached. The device broke under a stress of $39.5 \mathrm{MPa}$ at a strain of $17.4 \%$, which is consistent with the tensile results from the PNB-only samples.

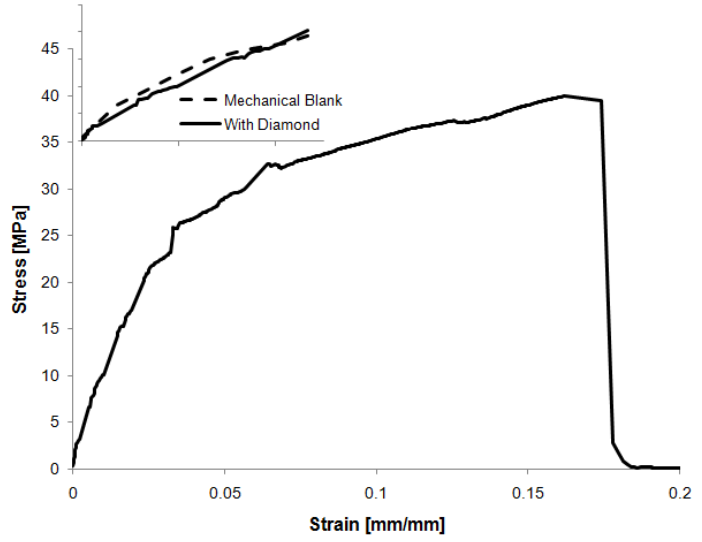

Figure 5: Stress-strain tensile plot for PNB tensile device with eight $100 \times 100 \mu^{2}$ diamond contacts down the length; (inset) comparison of slope of sample with diamonds to PNB-only sample.

The inset in Fig. 5 shows that in the linear regime, the diamond-based device behaves very similarly to a polymer-only device in terms of stiffness. Although the Young's modulus of diamond is very high $(\sim 1000 \mathrm{GPa})$, and the Young's modulus of the polymer is several orders of magnitude lower, the small diamond segments spaced relatively far apart allow the overall device to retain its flexibility and behave as a low-modulus structure. Further, upon breaking, the devices were inspected under an optical microscope. No evidence of diamond cracking or delamination was observed, suggesting that even at a strain of $17.4 \%$, the adhesion between the diamond and polymer was strong.

\section{ADHESION}

Adhesion of the diamond to the polymer substrates was evaluated using a qualitative adhesive-tape test. A piece of pressure-sensitive tape was pressed onto a tensile device with eight diamond electrodes, similar to the devices in Figure 4. The tape was then pulled up from the device, and both the tensile structure and tape were inspected. No delamination of the diamond pad from the polymer substrate was seen. The tape test was also performed on metalized diamond-based structures. It was observed that some of the metal was transferred to the tape, indicating that the adhesion between the metal and polymer is not as strong as between the polymer and diamond, indicating that a method to improve adhesion between the metal and base polymer is required.

\section{ELECTRICAL CHARACTERIZATION}

Electrical testing was performed in solution to evaluate the quality of the diamond electrodes as well as the integrity of the metal/diamond interfaces. Impedance spectra for a cortical electrode and a single square electrode from one of the 10 electrode arrays are shown in Figure 6. The electrode impedance at $1 \mathrm{kHz}$ is an important indicator of the ability of the electrode to record neural signals. The cortical electrode had an impedance of $2.8 \mathrm{M} \Omega$, and the array electrode had an impedance of $2.3 \mathrm{M} \Omega$ at 1 $\mathrm{kHz}$. These data are comparable to Au electrodes used in neural recording [10], suggesting that the inverted diamond electrodes would be able to record neural action potentials. Compared to $\mathrm{Au}$ electrodes, the impedance is relatively independent of frequency, resulting from the dominant resistive component of the impedance at low frequencies. A high-resistance metal-diamond contact or the 
diamond nucleation surface contributing to a low capacitance at the electrode-electrolyte interface may lead to the large resistive component of the electrode impedance.

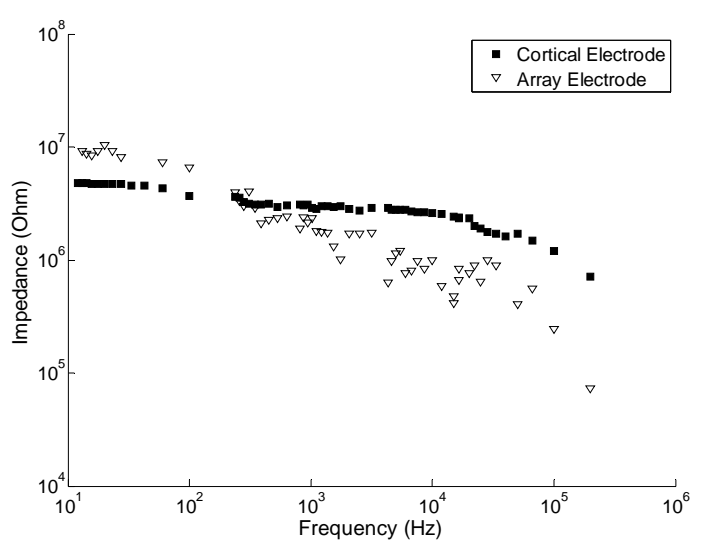

Figure 6: Impedance spectrum of a diamond-on-polymer electrode structure.

The electrodes were characterized for neurostimulation by applying charge-balanced, biphasic $1 \mathrm{~mA}, 100 \mu$ s current pulses generated by a multichannel stimulator at $5 \mathrm{~Hz}$, similar to those used in clinical prosthetic applications. A typical measured response for a single, $64 \times 64 \mu \mathrm{m}^{2}$ electrode is shown in Figure 7. The electrode voltage is superimposed onto the input signal as measured across a $1 \mathrm{k} \Omega$ resistor. The voltage drop was approximately $7 \mathrm{~V}$, which corresponds to an electrode resistance of $\sim 7 \mathrm{k} \Omega$ for a $1 \mathrm{~mA}$ signal. The access voltage was measured to be approximately $4.2 \mathrm{~V}$ and the electrode-electrolyte polarization is $2.8 \mathrm{~V}$ at a current density of $24 \mathrm{~A} \mathrm{~cm}^{-2}$. The ability to charge, and then discharge, the electrode-electrolyte interface and remain within the compliance voltage of the stimulator suggests that these electrodes can be used for neurostimulation applications.

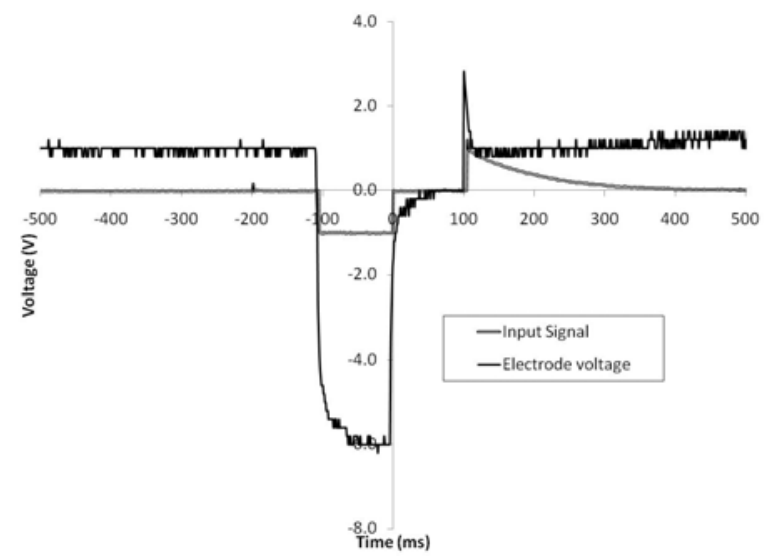

Figure 7: Voltage waveform recorded from diamond electrode during biphasic current pulse testing.

\section{ELECTROCHEMICAL CHARACTERIZATION}

Cyclic voltammetry was used to evaluate the use of the diamond-on-polymer electrodes for electrochemical sensing. It was found that there is a significant resistance inherent in the system, greatly reducing the sensitivity of the diamond as a sensor. There are a number of possible causes of the high resistance. The highly disordered nucleation surface of the diamond may be inappropriate for electrochemical sensing, or may be inadequately doped for good signal transduction. Alternatively, the contact resistance between the metal and diamond may be too high. Because the first polymer layer enters the fabrication process prior to metal deposition, an anneal step cannot be performed without modifying the fabrication process.

\section{CONCLUSION}

Mechanically-flexible, microfabricated, polycrystalline diamond-on-polymer electrode arrays have been fabricated using a novel transfer technique that avoids the problems associated with high-temperature diamond growth. Cortical probe electrodes and larger electrode arrays were fabricated to demonstrate the application of the fabrication process to a range of sizes and geometries. Mechanical testing has shown that the diamond-onpolymer structures are flexible despite the integration of the highly rigid diamond electrodes. Electrical characterization suggests that these electrodes may be useful in neural recording and stimulation applications. Further investigation is required to determine whether the inverted diamond electrodes can be used for chemical sensing.

\section{ACKNOWLEDGEMENTS}

This work was funded by grants from NIH (EB004018) and NSF (CTS 0449098) and (ECS 0621984). The authors thank Promerus, Inc. of Brecksville $\mathrm{OH}$ for providing Avatrel ${ }^{\mathrm{TM}}$.

\section{REFERENCES}

[1] S.F. Cogan, "Neural Stimulation and Recording Electrodes", Annual Review of Biomed. Engineering, 10 (2008) 275-309.

[2] H-Y Chan, D.M. Aslam, J.A. Wiler, B. Casey, "A Novel Diamond Microprobe for Neuro-chemical and -Electrical Recording in Neural Prosthesis", JMEMS, 18 (2009) 511-522.

[3] H.B. Martin, A. Argoitia, U. Landau, A.B. Anderson, J.C. Angus, "Hydrogen and Oxygen Evolution on Boron-Doped Diamond Electrodes", J. Electrochem. Soc., 143 (1996) L133L136.

[4] J.M. Halpern, S. Xie, G.P. Sutton, B.T. Higashikubu, C.A. Chestek, H. Lu, H.J. Chiel, H.B. Martin, "Diamond electrodes for neurodynamic studies in Aplysia californica", Diamond and Related Materials, 15, 183 (2006).

[5] T. Rao, A. Fujishima, "Recent advances in electrochemistry of diamond", Diamond and Rel. Mat., 9 (2000) 384-389.

[6] E. Popa, H.Notsu, T. Miwa, D.A. Tryk, A. Fujishima, "Selective Electrochamical Detection of Dopamine in the Presence of Ascorbic Acid at Anodized Diamond Thin Film Electrodes", Electrochem. Solid-State Lett, 2 (1999) 49-51.

[7] B.V. Sarada, T.N. RAo, D.A. Tryk, A. Fujishima, "Electrochemical oxidation of Histamine and Serotonin at Highly Boron-Doped Diamond Electrodes", Anal. Chem., 72 (2000), 1632-1638.

[8] P. Ariano, A.L. Gludice, A. Marcantoni, E. Vittone, E. Carbne, D. Lovisolo, "A diamond-based biosensor for the recording of neuronal activity", Biosensors and Bioelectronics, 24 (2009) 2046-2050.

[9] A Hess, J. Dunning, J. Harris, J.R. Capadona, K.Shanmuganathan, S.J. Rowan, C. Weder, D.J. Tyler, C.A. Zorman, "A Bio-inspired, Chemo-Responsive Polymer Nanocomposite for Mechanically Dynamic Microsystems", Transducers Conference, Denver, CO, June 21-25, 2009.

[10] N.A. Blum, B.G. Carkhuff, H.J. Charles Jr., R.L. Edwards, R.A. Meyer, "Multisite microprobes for neural recordings", IEEE Trans. Biomed. Eng., 38, 68 (1991).

\section{CONTACT}

*C.A. Zorman, tel: +1-216-368-6117; caz@,case.edu 